\title{
CHARACTERISTIC AND ORDER OF DIFFERENTIABLE POINTS IN THE CONFORMAL PLANE( $\left.{ }^{(}\right)$
}

\author{
BY \\ N. D. LANE AND P. SCHERK
}

Introduction. In [3], the authors discussed a definition of the conformal differentiability of an arc at a point in the conformal plane. It was based on the postulation of tangent circles and of an osculating circle. The intersection and support properties of all the circles through a differentiable point were studied, and by means of these properties the differentiable points were classified into various types. Each type was uniquely described by a certain triple of numbers, the characteristic of that type.

In this paper, relationships between the characteristic of a differentiable point and its cyclic order are established. In this connection some-partly familiar-differentiability properties of arcs of order three will be discussed. Our main results are stated in five theorems; cf. $\S \S 2.1,3.4,3.5,5.1,5.9$.

\section{Prerequisites}

1.1. In the following, $P, Q, \cdots$ denote points in the real conformal plane, and $C, C^{\prime}, \ldots$ denote oriented circles. Such a circle $C$ decomposes the plane into two open regions, its interior $C_{*}$ and its exterior $C^{*}$, the latter lying at its right. If $C$ degenerates into a point, then $C_{*}$ is empty. The circle through three mutually distinct points $P, Q$, and $R$, will occasionally be denoted by $C(P, Q, R)$.

The set of all circles that intersect two given circles at right angles forms a linear pencil $\pi$. A pencil $\pi$ of the first kind possesses two fundamental points. It consists of all the circles through these points. A pencil $\pi$ of the second kind has one fundamental point and is the set of those circles that touch a given circle at that point. [If $\pi$ is of the third kind, then any two circles of $\pi$ are disjoint.] For any pencil $\pi$ and for any point $Q$ which is not a fundamental point of $\pi$, there is a circle $C(\pi, Q)$ of $\pi$ through $Q$. It is unique except for its orientation. We consider the fundamental point of a pencil $\pi$ of the second kind a point-circle belonging to $\pi$.

1.2. We call a sequence of points $P_{1}, P_{2}, \cdots$ convergent to $P$ if to every circle $C$ with $P \subset C_{*}$ there corresponds a number $n=n(C)$ such that $P_{\lambda} \subset C_{*}$ if $\lambda>n$. In the same way the convergence of circles to a point is defined.

Presented to the Society, September 5, 1953; received by the editors July 25, 1955.

(1) This paper was prepared while the authors were Fellows at the Summer Research Institute of the Canadian Mathematical Congress. 
Suppose $C$ is not a point-circle. Then we call the sequence $C_{1}, C_{2}, \cdots$ convergent to $C$ if to every pair $C^{\prime} \subset C_{*}$ and $C^{\prime \prime} C C^{*}$ there corresponds a number $n=n\left(C^{\prime}, C^{\prime \prime}\right)$ such that $C^{\prime} \subset C_{* \lambda}$ and $C^{\prime \prime} \subset C_{\lambda}^{*}$ for every $\lambda>n$.

1.3. An $\operatorname{arc} A$ is defined as the continuous image of an interval. The same small italics-except $a$ and $n$-will be used to denote both the parameters, i.e., the points of the parameter interval, and their images on $A$. The end points [interior points] of $A$ are the images of the end points [interior points] of the parameter interval.

A neighbourhood of $p$ on $A$ is the image of a neighbourhood of the parameter $p$ on the parameter interval. If $p$ is an interior point of $A$, this neighbourhood is decomposed by $p$ into two (open) one-sided neighbourhoods.

From our definition, different points of $A$, i.e., points with different parameters, may coincide with the same point of the conformal plane. However, we shall assume that each point $p$ of $A$ has a neighbourhood such that no other point of that neighbourhood coincides with $p$. (The notation $s \neq p$ will indicate that the points $s$ and $p$ do not coincide.)

1.4. Suppose $p$ is an interior point of $A$. Then we call $p$ a point of support [intersection] with respect to the circle $C$ if some neighbourhood of $p$ is decomposed by $p$ into two one-sided neighbourhoods which lie in the same region [in different regions] bounded by $C$. $C$ is then called a supporting [intersecting] circle of $A$ at $p$. Thus $C$ supports $A$ at $p$ if $p \subset C$. By definition, the point-circle $p$ always supports $A$ at $p$.

It can happen that every neighbourhood of $p$ has points in common with $C$. Then $C$ neither supports nor intersects $A$ at $p$.

1.5. A point $p$ on $A$ is said to be (conformally) differentiable if it satisfies two conditions:

Condition I. For every point $R \neq p$, and for every sequence of points $s \rightarrow p$, $s \subset A, s \neq p$, there exists a circle $C_{0}$ such that $C(s, p, R) \rightarrow C_{0}$.

We call $C_{0}$ the tangent circle of $A$ at $p$ through $R$. Condition I implies [3]:

(i) There is a unique tangent circle $C_{0}=C(\tau, R)$ through each point $R \neq p$ and the union $\tau=\tau(p)$ of the set of tangent circles with the point circle $p$ is a pencil of the second kind with the fundamental point $p$. In particular, the angle between any two tangent circles is zero.

(ii) Nontangent circles through $p$ all intersect or all support.

(iii) Let $\pi$ be a pencil of the second kind with the fundamental point $p$; $\pi \neq \tau$. Then $\lim _{s \rightarrow p, s \neq p} C(\pi, s)=p$.

Condition II. If $s \rightarrow p, s \neq p$, there exists a circle $C(p)$ such that $C(\tau, s)$ $\rightarrow C(p)$.

We call $C(p)$ the osculating circle of $p . C(p)$ may be the point-circle $p$. Differentiability implies:

(iv) The nonosculating tangent circles through $p$ all intersect or all support. If $C(p) \neq p$, all of them support.

(v) $C(\pi, p)=\lim _{s \rightarrow p, s \neq p} C(\pi, s)$ exists for every $\pi$. 
1.6. In [3] the differentiable interior points $p$ of $A$ were classified into ten types according to the behaviour of the circles through $p$. We associated with $p$ a characteristic $\left(a_{0}, a_{1}, a_{2}\right)$ if $C(p) \neq p$, or $\left(a_{0}, a_{1}, a_{2}\right)_{0}$ if $C(p)=p$. The numbers $a_{0}$ and $a_{1}$ are equal to 1 or 2 , while $a_{2}$ is equal to 1,2 , or $\infty$. They have the following properties: $a_{0}\left[a_{0}+a_{1}\right]$ is even or odd according as the nontangent circles [the nonosculating tangent circles] of $p$ support or intersect; $a_{0}+a_{1}+a_{2}$ is even if $C(p)$ supports, odd if $C(p)$ intersects, while $a_{2}=\infty$ if $C(p)$ neither supports nor intersects. Thus $a_{0}+a_{1}+a_{2}$ is even if $C(p)=p$. From 1.5 (iv), $a_{0}=a_{1}$ if $C(p) \neq p$.

1.7. An $\operatorname{arc} A$ is said to be of finite cyclic order if it has only a finite number of points in common with any circle. If the least upper bound of these numbers is finite, then it is called the (cyclic) order of $A$, and $A$ is said to be of bounded cyclic order. The order of a point $p$ of $A$ then is the minimum of the orders of all the neighbourhoods of $p$ on $A$.

\section{THE ORDER OF A DIFFERENTIABLE POINT}

2.1. In this section we shall prove the following:

TheOREм 1. Let $p$ be a differentiable interior point of the arc $A$. Suppose that $p$ has the characteristic $\left(a_{0}, a_{1}, a_{2}\right)$ or $\left(a_{0}, a_{1}, a_{2}\right)_{0}$. Then the order of $p$ is not less than $a_{0}+a_{1}+a_{2}$.

The proof of this theorem will yield the following:

COROLLARy. If the order of $p$ is bounded, then there exists for every neighbourhood of $p$ a circle arbitrarily close to $C(p)$ which does not pass through $p$ and which intersects that neighbourhood in not less than $a_{0}+a_{1}+a_{2}$ points; $c f .1 .4$.

2.2. Let $B$ be an arc of finite order. If a circle $C$ intersects $B$ at $s$, then every circle sufficiently close to $C$ intersects $B$ in at least one point.

Proof. The end points of some neighbourhood $M \subset B$ of $s$ lie on opposite sides of $C$. Hence they also lie on opposite sides of any circle $C^{\prime}$ sufficiently close to $C$. Since $M$ and $C^{\prime}$ have only a finite number of points in common, one of them must be an intersection.

We note that $C^{\prime}$ will intersect $M$ in an odd number of points.

2.3. Let $\pi_{2}=\tau$ be the pencil of the tangent circles of $p$; thus $C\left(\pi_{2}, p\right)$ $=C(p)$. Let $\pi_{1}$ be any pencil of the first kind such that $p$ is one of its fundamental points and $C\left(\pi_{1}, p\right) \neq C(p)$. [Obviously, $C\left(\pi_{1}, p\right)$ is the tangent circle through the second fundamental point of $\pi_{1}$.] Finally, let $\pi_{0}$ be a pencil of the first kind such that $C\left(\pi_{0}, p\right) \llbracket \tau$. Let $M$ be a neighbourhood of $p$ on $A$. We wish to show that $\pi_{i}$ contains circles $C$ arbitrarily close to but different from $C_{i+1}=C\left(\pi_{i}, p\right)$ which meet $M$ outside $p$ in not less than $a_{i}$ points. If $p$ has finite order and if $M$ is small enough, then $C$ can be chosen such that the number of intersections of $M$ with $C$ exceeds $a_{i}$ by a non-negative even integer $\left[i=0,1,2\right.$; we assume $a_{2}<\infty$ in the case $\left.i=2\right]$. 
2.31. Let $D_{i} \subset \pi_{i}, D_{i} \neq C_{i+1}(i=0,1,2)$. If $i=2$, let $E_{2}=C_{3}$ when $C_{3} \neq p$, but let $E_{2}=D_{2}$ when $C_{3}=p$. If $i<2, E_{i}$ will not be defined. However, we still define the regions

$$
E_{* i}=\left(C_{* i+1} \cap D_{i}^{*}\right) \cup\left(C_{i+1}^{*} \cap D_{* i}\right)
$$

and

$$
E_{i}^{*}=\left(C_{* i+1} \cap D_{* i}\right) \cup\left(C_{i+1}^{*} \cap D_{i}^{*}\right) \quad \text { [cf. Figure 1]. }
$$

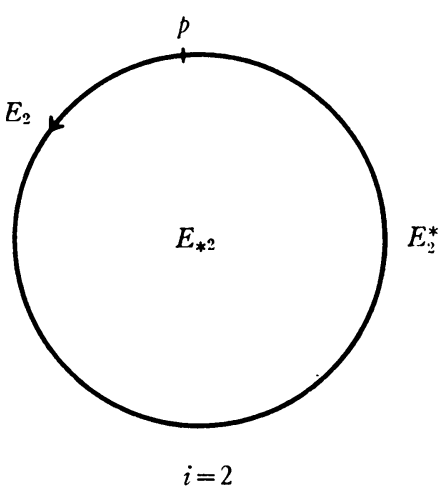

$i=2$

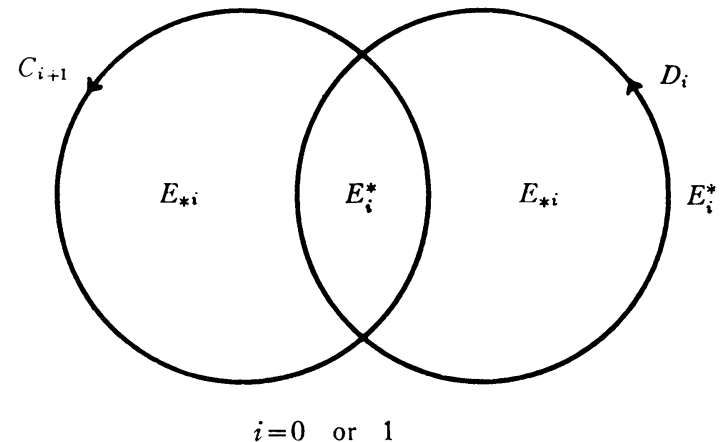

FIG 1.

Let $\pi_{*_{i}}\left[\pi_{i}^{*}\right]$ denote the set of those circles of $\pi_{i}$ that pass through $E_{*_{i}}\left[E_{i}^{*}\right]$. Then every circle of $\pi_{i}$ except $C_{i+1}$ and $D_{i}$ belongs either to $\pi_{* i}$ or to $\pi_{i}^{*}$. By intersecting $\pi_{i}$ with an orthogonal circle, we can construct a continuous oneto-one correspondence between the circles of $\pi_{* i}\left[\pi_{i}{ }^{*}\right]$ and the points of an interval and hence a "betweenness" relation in $\pi_{*_{i}}\left[\pi_{i}{ }^{*}\right]$.

We can choose our neighbourhood $M$ so small that $C_{i+1}$ and $D_{i}$ have no points in common with the two one-sided neighbourhoods $N$ and $N^{\prime}$ into which $M$ is decomposed by $p$. This follows for $C_{3}$ from our assumption $a_{2}<\infty$, and for the other circles from 1.5. Thus $N\left[N^{\prime}\right]$ lies entirely in one of the two regions $E_{* i}$ and $E_{i}^{*}$. Let $s$ and $s^{\prime}$ denote the points of $N$ and $N^{\prime}$ respectively. Thus either all the circles $C\left(\pi_{i}, s\right)$ belong to $\pi_{* i}$, or all of them are in $\pi_{i}^{*}$. Without restriction of generality, let $N \subset C_{i+1}^{*} \cap D_{i}^{*} \subset E_{i}^{*}$. Then $C\left(\pi_{i}, s\right) \subset \pi_{i}^{*}$ for every $s$.

2.32. Let $e \subset N$. Then $C\left(\pi_{i}, e\right)$ is the end-circle of a one-sided neighbourhood $\nu$ of $C_{i+1}$ in $\pi_{i}$. If $s$ moves from $e$ to $p$, then $C\left(\pi_{i}, s\right)$ moves in $\pi_{i}$ from $C\left(\pi_{i}, e\right)$ continuously to $C_{i+1}$. Hence the circles $C\left(\pi_{i}, s\right)$ omit none of the circles of $\nu$; i.e., every circle of $\nu$ meets $N$.

Let $C \subset \nu$. Thus $C$ lies between $C\left(\pi_{i}, e\right)$ and $C_{i+1}=\lim _{s \rightarrow p} C\left(\pi_{i}, s\right)$. If $s$ lies sufficiently close to $p$, then $s \nsubseteq C$ and $C$ will also lie between $C\left(\pi_{i}, e\right)$ and $C\left(\pi_{i}, s\right)$. Since $e \nsubseteq \subset C$ and since the points $s$ and $e$ lie in $C_{i+1}^{*} \cap D_{i}^{*}$, they will also be separated by $C$. 
Let the order of $p$ be finite. Then we may assume that $M$ also is of finite order. In particular, $C$ will meet $N$ in a finite number of points only, and at least one of them will be an intersection. Replacing $N$ by the one-sided neighbourhood of $p$ with the end-point $e$, we can even state that $C$ will intersect $N$ in an odd number of points.

Similarly, there exists a one-sided neighbourhood $\nu^{\prime}$ of $C_{i+1}$ in $\pi_{i}$ such that each of its circles meets $N^{\prime}$. If $p$ has finite order and if $N^{\prime}$ is sufficiently small, then $\nu^{\prime}$ can be chosen such that each circle of $\nu^{\prime}$ intersects $N^{\prime}$ in an odd number of points.

2.33. If $a_{i}=1$, then one of the circles $C_{i+1}$ and $D_{i}$ intersects while the other one supports $M$ at $p$; therefore $N^{\prime} \subset E_{*_{i}}$. If $a_{i}=2$, then $C_{i+1}$ and $D_{i}$ either both intersect or both support; hence $N^{\prime} \subset E_{i}{ }^{*}$. Thus the circles $C\left(\pi_{i}, s^{\prime}\right)$ belong to $\pi_{* i}\left[\pi_{i}{ }^{*}\right]$ if $a_{i}=1[=2]$. This holds true, in particular, of the circles of the neighbourhood $\nu^{\prime}$ constructed in 2.32. Since $\nu \subset \pi_{i}^{*}$, it follows that $\nu$ and $\nu^{\prime}$ lie on opposite sides of $C_{i+1}$ or on the same side depending on whether $a_{i}=1$ or $=2$. This implies our statements 2.3 .

2.4 . We can now readily verify the assertions of 2.1 . Obviously, we may assume that the order of $p$ is finite; in particular, $a_{2}<\infty$.

We prove our theorem by first approximating $C(p)$ by a tangent circle, then the latter by a nontangent circle through $p$, and finally that circle by one which does not contain $p$.

Let $M_{2}$ be a neighbourhood of finite order of $p$ on $A$. From 2.3 there exists a circle $C_{2} \subset \tau$, close to but different from $C(p)$, which intersects $M_{2}$ in not less than $a_{2}$ points $s_{2}$ outside $p$.

In $M_{2}$ we construct mutually disjoint neighbourhoods $B_{2}$ of the points $s_{2}$ and $M_{1}$ of $p$. Let $\pi_{1}$ be the pencil of the circles through $p$ and another point of $C_{2}$; thus $C_{2}=C\left(\pi_{1}, p\right)$. From 2.3 and 2.2 there is a circle $C_{1} \subset \pi_{1}$, close to but different from $C_{2}$, which intersects $M_{1}$ in not less than $a_{1}$ points $s_{1}$ outside $p$ and which also intersects each $B_{2}$.

Finally, construct in $M_{\mathrm{i}}$ mutually disjoint neighbourhoods $B_{1}$ about each $s_{1}$ and $M_{0}$ about $p$. Let $\pi_{0}$ be the pencil of the first kind through two points $\not p$ of $C_{1}$; thus $C_{1}=C\left(\pi_{0}, p\right)$. From 2.3 and 2.2 there is a circle $C_{0} \subset \pi_{0}$, close to but different from $C_{1}$, which intersects $M_{0}$ in not less than $a_{0}$ points outside $p$ and which intersects each of the $a_{1}+a_{2}$ arcs $B_{1}$ and $B_{2}$.

Altogether, $C_{0}$ will be close to $C(p)$ and intersect $M_{2}$ in not less than $a_{0}+a_{1}+a_{2}$ points all of which are different from $p$.

\section{Differentiability PROPERTIES OF ARCS OF ORDER THREE}

3.1. We call $C$ a general tangent circle of an $\operatorname{arc} A$ at a point $p$ if there exists a sequence of triplets of mutually distinct points $u, v, Q$ such that $u$ and $v$ converge on $A$ to $p$ and that

$$
\lim C(u, v, Q)=C .
$$


If, in addition, $Q \subset A$ also converges to $p$, then we call $C$ a general osculating circle of $A$ at $p$.

We call $A$ strongly differentiable at $p$ if the following conditions are satisfied:

Condition I'. Let $R \neq p, Q \rightarrow R$. If two distinct points $u$ and $v$ converge on $A$ to $p$, then $C(u, v, Q)$ always converges.

Condition II'. $C(t, u, v)$ converges if the three mutually distinct points $t, u v$ converge on $A$ to $p$.

The first condition implies that the limit circle (3.1) depends on $p$ and $R$ but not on the choice of the particular sequences $u$ and $v$. Specializing $Q=R$ and $u=p$, we see that Condition $\mathrm{I}^{\prime}$ implies Condition I and that therefore

$$
\lim C(u, v, Q)=C(\tau, R) .
$$

Thus the general tangent circles of a point which satisfies Condition $I^{\prime}$ are identical with its ordinary ones.

Suppose $A$ is strongly differentiable at $p$. From the above, Condition I will hold at $p$. Suppose a sequence of points $u$ converges on $A$ to $p$. We may assume that the circles $C(\tau, u)$ converge. Each of them can be approximated by a circle $C(p, u, v)$ with the same limit circle and such that the sequence $v$ also converges to $p$. On account of Condition $\mathrm{II}^{\prime}, \lim C(t, u, v)$, and in particular $\lim C(p, u, v)$, is independent of the choice of the sequences $t, u, v$ converging to $p$. Hence the same will hold true of $\lim C(\tau, u)$, Condition II is satisfied, and we have

$$
\lim _{\imath, u, v \rightarrow p} C(t, u, v)=\lim _{u, v \rightarrow p} C(p, u, v)=\lim _{u \rightarrow p} C(\tau, u)=C(p) .
$$

Thus strong differentiability implies ordinary differentiability and $C(p)$ is the one and only general osculating circle.

There are other extensions of the above conditions to cases in which some of the points involved coincide. The reader will readily verify them along the lines of the preceding proof.

(i) Let $p$ satisfy Condition $I^{\prime}$. Let $R \neq p, Q \rightarrow R$. Let $u$ converge on $A$ to $p$ and let $C_{1}$ be a general tangent circle at u through $Q$. Then $\lim C_{1}=C(\tau, R)$.

(ii) Suppose $A$ is strongly differentiable at p. Let the two distinct points $u$ and $v$ converge on $A$ to $p$ and let $C_{2}$ denote a general tangent circle at $u$ through $v$. Then $\lim C_{2}=C(p)$; cf. (3.3).

(iii) Suppose $A$ is strongly differentiable at $p$. Let u converge on $A$ to $p$ and let $C_{3}$ be a general osculating circle at $u$. Then $\lim C_{3}=C(p)$.

3.2. Let $p$ be an end point of the arc $A$ of finite cyclic order. It is well known that $A$ is differentiable at $p$; cf. [2]. For the reader's convenience, we include a proof.

Suppose Condition I of 1.5 is not satisfied. Then for some point $R \neq p$ there are two sequences of points $s_{2 k}$ and $s_{2 k+1}$ different from $p$ and converging on $A$ to $p$ such that the circles $C_{2 k}=C\left(s_{2 k}, p, R\right)$ and $C_{2 k+1}=C\left(s_{2 k+1}, p, R\right)$ con- 
verge to different limit circles $C_{0}$ and $C_{1}$ respectively. We may assume that $s_{n+1}$ lies between $p$ and $s_{n}$. If $k$ is large, $C_{2 k}\left[C_{2 k+1}\right]$ will lie close to $C_{0}\left[C_{1}\right]$. Let $C$ and $C^{\prime}$ be two circles through $p$ and $R$ which separate $C_{0}$ and $C_{1}$. Then $C \cup C^{\prime}$ will separate $C_{n}$ and $C_{n+1}$ and therefore also $s_{n}$ and $s_{n+1}$ for every large $n$. Hence the sub-arc of $A$ bounded by $s_{n}$ and $s_{n+1}$ will meet $C \cup C^{\prime}$ in at least one point. Thus $A$ will meet $C \cup C^{\prime}$ infinitely often. This is impossible.

Similarly, the validity of Condition II can be verified.

3.3. In the following, let $A_{3}$ denote an open arc of order three. We readily verify that a point of $A_{3}$ converges if its parameter tends to one of the end points of the parameter interval. Thus $A_{3}$ has two well-defined end points. Let $p$ denote one of them.

We introduce multiplicities counting $p$ [a point $q$ of $A_{3}$ ] three times on $C(p)$ [on a general osculating circle at $q$ ] and twice on any other [general] tangent circle of $A_{3}$ at $p$ [at $q$ ]. We wish to show that no circle meets $A_{3} \cup p$ more than three times, i.e., the inclusion of $p$ and the introduction of multiplicities do not alter the order of $A_{3}$.

Obviously, any circle through $q$ will either support or intersect $A_{3}$ there. A general osculating circle at $g$ intersects there while any other general tangent circle supports. Conversely, any supporting circle is a general tangent circle.

3.31. Suppose a circle $C$ through $p$ intersects $A_{3}$ at $q$ and meets $A_{3}$ in two more points $r$ and $s$. Choose disjoint neighbourhoods $N$ of $p$ and $M$ of $q$ on $A_{3}$ which do not contain $r$ or $s$. If $v$ converges in $N$ to $p$, then $C(r, s, v)$ converges to $C$. From 2.2, $C(r, s, v)$ will intersect $M$ if $v$ is sufficiently close to $p$. Thus this circle meets $A_{3}$ in not less than four points. This yields

Lemma 1. If a circle through $p$ meets $A_{3}$ at three points, then all of them are points of support.

Similarly, if a tangent circle of $p$ intersects $A_{3}$ at $q$ and meets $A_{3}$ also at $r \neq q$, then there will be a circle through $p$ and $r$ which intersects $A_{3}$ near $q$ and also meets $A_{3}$ near $p$. By Lemma 1 this is impossible. This implies

Lemma 2. If a tangent circle of $p$ meets $A_{3}$ at two points, then both of them are points of support.

In the same way, Lemma 2 finally implies

Lemma 3. $C(p)$ does not intersect $A_{3}$.

3.32. If a circle supports $A_{3}$ at $q$ and also meets $A_{3} \cup_{p}$ at $r$ and $s$, then a suitable circle near it through $r$ and $s$ intersects $A_{3}$ twice near $q$. By Lemma 1 and the definition of $A_{3}$ this is impossible. Hence a circle through three points of $A_{3} \cup p$ does not support $A_{3}$ at any of them.

Combining this result with Lemma 1 we obtain: No circle meets $A_{3} \cup p$ in four points. 
Similarly, if a tangent circle of $p$ supports $A_{3}$ at $q$, then there is near it another tangent circle of $p$ which intersects $A_{3}$ twice near $q$. This is excluded by Lemma 2 . Thus no tangent circle of $p$ supports $A_{3}$.

Applying the last result to Lemma 2, we have: No tangent circle of $p$ meets $A_{3}$ in more than one point.

The above and Lemma 3 imply that $C(p)$ does not meet $A_{3}$.

3.33. Suppose $C$ supports $A_{3}$ at two distinct points $q$ and $r$. From 3.32, $C \cap A_{3}=g \cup r$. Hence $A_{3} \subset C \cup C^{*}$, say. Let $M$ and $N$ be two disjoint neighbourhoods on $A_{3}$ of $q$ and $r$ respectively. Choose a circle $D$ in $C^{*}$ and sufficiently close to $C$; cf. 1.2. Since the end points of $M$ and $N$ lie in $C^{*}$, they will also lie in $D^{*}$. On the other hand $C \subset D_{*}$ implies $q \subset D_{*}$ and $r \subset D_{*}$. Thus $D$ separates $q[r]$ from the end points of $M[N], D$ will intersect $M[N]$ in not less than two points, and $D \cap A_{3}$ contains more than three points.

3.34. Let $C$ be a general osculating circle of $A_{3}$ at an interior point $q$. Thus $C=\lim C\left(q_{n}, q_{n}^{\prime}, q_{n}^{\prime \prime}\right)$ where the three mutually distinct points $q_{n}, q_{n}^{\prime}$, $q_{n}^{\prime \prime}$, converge on $A_{3}$ to $q$.

Suppose $C$ meets $A_{3} \cup p$ at a point $r \neq q$. Then the normal circle of $C$ through $q$ and $r$ will intersect $C\left(q_{n}, q_{n}^{\prime}, q_{n}^{\prime \prime}\right)$ at a point $R_{n}$ converging to $r$. Thus

$$
C\left(q_{n}, q_{n}^{\prime}, q_{n}^{\prime \prime}\right)=C\left(q_{n}, q_{n}^{\prime}, R_{n}\right) .
$$

The circles $C\left(q_{n}, q_{n}^{\prime}, r\right)$ will not meet $A_{3} \cup p$ elsewhere and they will intersect $A_{3}$ at $q_{n}$ and $q_{n}^{\prime}$. Thus the end points of any small neighbourhood of $q$ will lie on the same side of $C\left(q_{n}, q_{n}^{\prime}, r\right)$ if $n$ is large enough. Hence any limit circle $D$ of $C\left(q_{n}, q_{n}^{\prime}, r\right)$ will support $A_{3}$ at $q$.

Let $Q_{1}, Q_{2}, S, T$ be variable points and let $Q_{1}$ and $Q_{2}$ converge to the same point $P ; Q_{1} \neq Q_{2}$. Suppose there is a fixed circle separating $P$ from both $S$ and $T$. Then

$$
\lim \Varangle\left[C\left(Q_{1}, Q_{2}, S\right), C\left(Q_{1}, Q_{2}, T\right)\right]=0
$$

whether the circles $C\left(Q_{1}, Q_{2}, S\right)$ and $C\left(Q_{1}, Q_{2}, T\right)$ themselves converge or not. In particular

$$
\lim \Varangle\left[C\left(q_{n}, q_{n}^{\prime}, R_{n}\right), C\left(q_{n}, q_{n}^{\prime}, r\right)\right]=0 .
$$

Since the angle between two circles depends on them continuously, it follows that $\Varangle(C, D)=0$. Since $C$ and $D$ have the points $q$ and $r$ in common, this implies $C=D$. However, $D$ supports and $C$ intersects $A_{3}$ at $q$. Hence $C$ does not meet $A_{3} \cup p$ outside $q$.

3.4. Let $A_{3}$ again denote an open arc of order three. The preceding subsections enable us to discuss the differentiability of $A_{3}$.

3.41. We first prove

Theorem 2. Every point of $A_{3}$ satisfies Condition $\mathrm{I}^{\prime} ; c f .3 .1$. 
Proof. Let $q \subset A_{3}, e \subset A_{3}, e \neq q$. Choose two disjoint one-sided neighbourhoods $N$ and $N^{\prime}$ of $q$ such that $e \nsubseteq M=N \cup q \cup N^{\prime}$. Let $C_{1}$ and $C_{2}$ denote two general tangent circles at $q$ through $e$. Thus $C_{i}$ meets $A_{3}$ at least twice at $q$ and altogether at least three times. Hence $C_{i}$ meets $A_{3}$ exactly twice at $q$, once at $e$ and nowhere else. In particular, $C_{i}$ supports $A_{3}$ at $q$. Without loss of generality we may assume $N \cup N^{\prime} \subset C_{* 1} \cap C_{* 2}$ (cf. 3.3).

Suppose $C_{1} \neq C_{2}$. Then there is a third circle $C_{3}$ through $q$ and $e$ which does not meet $C_{* 1} \cap C_{* 2}$. Thus $C_{3}$ will also support $A_{3}$ at $q$. We may assume $N \cup N^{\prime} \subset C_{* 3}$.

By 3.2, the arcs $N \cup_{q}$ and $N^{\prime} \cup q$ satisfy Condition I. Thus they possess two well-defined tangent circles at $q$ through $e$. At least one of the circles $C_{1}, C_{2}, C_{3}$, say the circle $C$, is different from them. Let $\pi$ denote the pencil of the second kind of the circles touching $C$ at $q$.

Let $s \subset N \cup N^{\prime}$. Thus $s \subset C_{*}$ and hence $C(\pi, s) \subset C_{*} \cup q$. By 1.5, (iii), $\lim C(\pi, s)=q$ if $s$ approaches $q$ through $N$ or $N^{\prime}$. Since $C(\pi, s)$ depends continuously on $s$, there are circles in $\pi$ which are arbitrarily small and meet both $N$ and $N^{\prime}$ near $q$. Thus they meet $M$ not less than three times. On the other hand, the end points of $M$ will lie on the same side of such a small circle. Hence it will meet $M$ with an even multiplicity and therefore not less than four times. This being impossible we obtain $C_{1}=C_{2}$. Thus the general tangent circle at $q$ through $e$ is unique.

Choose $R, Q, u, v$ according to Condition $\mathrm{I}^{\prime}$. Then by (3.4),

$$
\lim \Varangle[C(u, v, Q), C(u, v, e)]=0 .
$$

Thus any limit circle of $C(u, v, Q)$ touches the general tangent circle at $q$ through $e$ at the point $q$. It also passes through $R$. Hence it is uniquely determined, q.e.d.

3.42. Continuing the preceding discussion we now study the general osculating circles of $A_{3}$ at $q$.

By 3.41 and 3.1, $A_{3}$ satisfies Condition I. The set of the general tangent circles of $A_{3}$ is identical with that of its ordinary ones. By 1.5, (i), this set is a pencil $\tau$ of the second kind with the fundamental point $q$. It is identical in particular with the pencil of the tangent circles of $N \cup q$ at $q$.

By 3.2, $N \cup_{q}\left[N^{\prime} \cup_{q}\right]$ has an osculating circle $C=C(q)\left[C^{\prime}=C^{\prime}(q)\right]$ at $q$. It belongs to $\tau$ and is readily seen to be a general osculating circle of $A_{3}$; cf. 3.1 .

Let $D$ denote any general osculating circle of $A_{3}$ at $q$. Thus $D \subset \tau$. From 3.3, $D$ intersects $A_{3}$ at $q$ and does not meet it elsewhere. Hence

$$
D \neq q
$$

and $D \neq C(\tau, e)$. We orient the circle $D$ such that $e \subset D^{*}$. If $e$ and $N$, say, lie on the same side of $q$, then

$$
N \subset D^{*} \text { and } N^{\prime} \subset D_{*} .
$$


Thus $s \subset N$ implies $C(\tau, s) \subset D^{*} \cup q$. Letting $s$ tend to $q$ we obtain

$$
C \subset D^{*} \cup D \text { and symmetrically } C^{\prime} \subset D_{*} \cup D \text {. }
$$

In particular $C \subset C^{* \prime} \cup C^{\prime}$ and $C^{\prime} \subset C_{*} \cup C$. Hence $C^{*} \subset C^{* \prime}$ and $C_{*}^{\prime} \subset C_{*}$ and therefore by (3.6),

$$
N \subset C^{*} \subset C^{* \prime} \text { and } N^{\prime} \subset C_{*} \subset C_{*} \text {. }
$$

By (3.5), $C \neq q$ and $C^{\prime} \neq q$. Thus there is a closed subinterval $\iota$ of $\tau$ bounded by $C$ and $C^{\prime}$ which does not contain the point-circle $q$. By (3.7), every general osculating circle belongs to $\iota$. Conversely by (3.8), every circle of $\iota$ separates $N$ and $N^{\prime}$. Thus it intersects $A_{3}$ at $q$. Being a general tangent circle it must be a general osculating circle. We thus have: The set of the general osculating circles at $q$ is equal to 1 . As a corollary we obtain the equivalence of the following properties:

(i) $C(q)=C^{\prime}(q)$,

(ii) $A_{3}$ is differentiable at $q$,

(iii) $A_{3}$ is strongly differentiable at $q$.

3.5. In the remainder of this section we shall prove

Theorem 3. Let $p$ be an end point of an open arc $A_{3}$ of order three. Then $A_{3} \cup p$ is strongly differentiable at $p ; c f .3 .1$ and 3.2.

We prepare our proof by a discussion which will also be useful further on.

3.51. Let $B$ be an open sub-arc of $A_{3}$ bounded by $p$ and any point $e$ of $A_{3}$. Let $d$ be any point of $A_{3}$ outside $B \cup e$. We orient the circles $C$ with $d \nsubseteq C$ such that $d \subset C^{*}$. The set of these circles contains all the circles which meet $A_{3} \cup_{p}$ three times in $p \cup B \cup e$. Their orientation is continuous. In particular the regions $C^{*}(\tau, u)$ and $C^{*}(t, u, v)$ depend continuously on $t, u, v$ when these points range through $p \cup B \cup e$ without all of them coinciding [cf. Theorem 2. Here and in the following, $\tau$ indicates the pencil of the tangent circles of $A_{3} \cup p$ at $\left.p\right]$.

Since $d \subset C^{*}(\tau, e)$ and $d \subset C^{*}(p)$, and since $C(\tau, e)$ intersects $A_{3}$ at $e$ while $C(p)$ does not meet $A_{3}$, we have

$$
u \subset C_{*}(\tau, e) \cap C^{*}(p) \quad \text { for every } u \subset B .
$$

Hence

$$
C(\tau, u) \subset\left[C_{*}(\tau, e) \cap C^{*}(p)\right] \cup p .
$$

Since $C(\tau, u)$ depends continuously on $u$, the following converse holds. Let $C \subset \tau, C \subset\left[C_{*}(\tau, e) \cap C^{*}(p)\right] \cup p$. Then there is a $u \subset B$ such that $C=C(\tau, u)$.

Formula (3.9) implies in particular

$$
C(\tau, u) \subset C_{*}(\tau, e) \cup p \text { and } C(\tau, u) \subset C^{*}(p) \cup p .
$$

Similarly 


$$
C(\tau, e) \subset C^{*}(\tau, u) \cup p .
$$

Also $C(\tau, t) \subset C_{*}(\tau, u) \cup p$ for every $t$ between $p$ and $u$. If $t$ tends to $p$, this yields $C(p) \subset C_{*}(\tau, u) \cup C(\tau, u)$ and hence

$$
C(p) \subset C_{*}(\tau, u) \cup p .
$$

On account of these formulas, (3.9) can be reformulated as follows:

$$
C_{*}(p) \subset C_{*}(\tau, u) \subset C_{*}(\tau, e) \quad\left[\text { thus } C^{*}(\tau, e) \subset C^{*}(\tau, u) \subset C^{*}(p)\right]
$$

for every $u \subset B$.

3.52. From now on, the points $t, u, v, e$ are assumed to be mutually distinct and to lie on $B \cup e$ in the indicated order. The circle $C(p, t, v)[C(t, v, e)]$ meets $A_{3} \cup p$ three times only and intersects $A_{3}$ at $v$ [and $\left.e\right]$. Hence $d \subset C^{*}(p, t, v)$ and $d \subset C^{*}(t, v, e)$ imply

$$
u \subset C_{*}(p, t, v) \cap C^{*}(t, v, e) .
$$

The circle $C(t, u, v)$ intersects $C(p, t, v)$ and $C(t, v, e)$ at $t$ and $v$. It is divided by $t$ and $v$ into two arcs. By (3.11) the arc that contains $u$ lies in

$$
C_{*}(p, t, v) \cap C^{*}(t, v, e) .
$$

Hence the other arc lies in

$$
C^{*}(p, t, v) \cap C_{*}(t, v, e)
$$

and we have

(3.12) $C(t, u, v) \subset\left[C_{*}(p, t, v) \cap C^{*}(t, v, e)\right] \cup\left[C^{*}(p, t, v) \cap C_{*}(t, v, e)\right] \cup t \cup v$. In particular $C(t, u, v)$ separates the regions

$$
C_{*}(p, t, v) \cap C_{*}(t, v, e)
$$

and

$$
C^{*}(p, t, v) \cap C^{*}(t, v, e) .
$$

The above argument remains valid in the case $t=p$ if we interpret $C(p, p, v)$ to mean $C(\tau, v)$. Thus

$$
C(p, u, v) \subset\left[C_{*}(\tau, v) \cap C^{*}(p, v, e)\right] \cup\left[C^{*}(\tau, v) \cap C_{*}(p, v, e)\right] \cup p \cup v
$$

and $C(p, u, v)$ separates

$$
C_{*}(\tau, v) \cap C_{*}(p, v, e)
$$

and

$$
C^{*}(\tau, v) \cap C^{*}(p, v, e) .
$$

The argument leading to (3.12) is also seen to remain valid if the points $v$ and $e$ are interchanged. Thus 


$$
C(t, u, e) \subset\left[C_{*}(p, t, e) \cap C^{*}(t, v, e)\right] \cup\left[C^{*}(p, t, e) \cap C_{*}(t, v, e)\right] \cup t \cup e .
$$

3.6. The proof of Theorem 3 naturally splits into two parts. In this subsection we show that the end point $p$ of $A_{3}$ satisfies Condition I'.

We first prove

$$
\lim _{u, 0 \rightarrow p} C(u, v, e)=C(\tau, e) .
$$

Let $D$ denote a limit circle of $C(t, u, e)$ as $t$ and $u$ tend to $p$. By (3.18) $D$ lies in

$$
\left[C_{*}(\tau, e) \cap C^{*}(p, v, e)\right] \cup\left[C^{*}(\tau, e) \cap C_{*}(p, v, e)\right] \cup \widetilde{C}(\tau, e) \cup C(p, v, e) .
$$

This holds for every choice of $v$ in $B$ while $D$ is independent of $v$. Letting $v$ tend to $p$, we obtain $D \subset C(\tau, e)$. Since $D$ passes through $p$ and $e$, this implies $D=C(\tau, e)$. Changing our notation we obtain (3.19).

Let $R, Q, u, v$ be defined according to Condition I'. We apply (3.4) with $P=p, Q_{1}=u, Q_{2}=v, S=e, T=Q$. Thus

$$
\lim _{u, v \rightarrow p, Q \rightarrow R} \Varangle[C(u, v, e), C(u, v, Q)]=0 .
$$

This relation and (3.19) imply: The circle $C(\tau, e)$ forms the angle zero with any limit circle of the circles $C(u, v, Q)$. Since such a circle contains $p$ and $R$, it is uniquely determined. This proves our statement.

3.7. Let $C_{1}=C(p, u, v)$ and $C_{2}=C(t, u, v)$. We prove simultaneously

$$
\lim _{u, v \rightarrow p} C_{1}=C(p)
$$

and assuming (3.20)

$$
\lim _{t, u, v \rightarrow p} C_{2}=C(p)
$$

Thus $p$ also satisfies Condition $\mathrm{II}^{\prime}$.

By (3.15) [by (3.12)], the circle $C_{1}\left[C_{2}\right]$ lies in

$$
C_{*}(\tau, v) \cup C_{*}(p, v, e) \cup p \cup v \quad\left[C_{*}(p, t, v) \cup C_{*}(t, v, e) \cup t \cup v\right]
$$

and it separates the regions (3.16) and (3.17) [(3.13) and (3.14)]. By 3.2, (3.20), and (3.19) we have

$$
\lim _{v \rightarrow p} C(\tau, v)=\lim _{t, v \rightarrow p} C(p, t, v)=C(p)
$$

and

$$
\lim _{o \rightarrow p} C(p, v, e)=\lim _{t, v \rightarrow p} C(t, v, e)=C(\tau, e) .
$$

Hence any limit circle $D_{i}$ of the circles $C_{i}$ has the following properties: 
(i) $D_{i}$ will lie in the closure of $C_{*}(p) \cup C_{*}(\tau, e)=C_{*}(\tau, e)$,

(ii) $D_{i}$ will separate the regions

$$
C_{*}(p) \cap C_{*}(\tau, e)=C_{*}(p) \text { and } C^{*}(p) \cap C^{*}(\tau, e)=C^{*}(\tau, e)
$$

unless one of them is void, i.e., unless $C(p)=p$; cf. (3.10).

Since $p \subset D_{i}$, (i) implies

$$
D_{i} \subset \tau \text {. }
$$

If $D_{i}$ is equal to $C(\tau, e)$ or passes through $C^{*}(p) \cup C_{*}(\tau, e)$, then it intersects $A_{3}$ at another point $r$ [cf. 3.51]. By 2.2, a circle $C_{i}$ sufficiently close to $D_{i}$ would meet $A_{3} \cup p$ three times near $p$ and also near $r$. Hence

$$
D_{i} \subset C_{*}(p) \cup C(p) \text {. }
$$

On account of (ii), this formula implies $D_{i}=C(p)$, whether $C(p)=p$ or not.

\section{LEMMAS ON ARCS OF ORDER THREE}

In this section we collect additional material on $\operatorname{arcs} A_{3}$ of order three needed in the last part of this paper. Let $p$ denote an end point of $A_{3}$. The arc $B$ and the points $d$ and $e$ are defined in 3.51. If $s \subset B$ and $P \neq s, B$ has a well-defined tangent circle $C(s, s, P)=C(P, s, s)$ at $s$ through $P$; cf. Theorem 2.

4.1. We first extend the formulas (3.12) and (3.15) of 3.52 to certain limit cases in which some of the points involved coincide.

4.11. If $t$ and $v$ are kept fixed while $u$ tends to $t[v]$, the right-hand sides of (3.12) and (3.15) are not affected while $C(t, u, v)$ tends to $C(t, t, v)[C(t, v, v)]$. Hence $C(t, t, v)$ and $C(t, v, v)[C(p, v, v)]$ lie in the closures of the regions given in (3.12) [(3.15)]. Since the circles which enter these formulas are mutually distinct, it follows that (3.15) [(3.12)] remains valid for $u=v$ [or $u=t]$.

We now keep $t$ and $u$ fixed. Then $C(t, u, v)$ and the regions of (3.12) and (3.15) depend continuously on $v$. Letting $v$ tend to $e$, we find, e.g., that $C(t, u, e)$ lies in the closure of

$$
\left[C_{*}(p, t, e) \cap C^{*}(t, e, e)\right] \cup\left[C^{*}(p, t, e) \cap C_{*}(t, e, e)\right] .
$$

But $C(p, t, e)$ and $C(t, e, e)$ are distinct from $C(t, u, e)$; cf. 3.3. Thus (3.12) and similarly (3.15) remain valid for $v=e$.

4.12. Let $s \subset B$ and let $C_{1}$ denote any general osculating circle of $B$ at $s$. Thus $C_{1}$ will be the limit of $C(t, u, v)$ if $t, u, v$ converge to $s$ in a suitable fashion. By Theorem 2 and 3.42, $C(p, t, v)[C(t, v, e)]$ then converges to $C_{2}=C(p, s, s)$ $\left[C_{3}=C(s, s, e)\right]$ and the circles $C_{1}, C_{2}, C_{3}$ touch at $s$. Furthermore $p \subset C_{* 3}$ and $e \subset C_{2}^{*}$ imply $C_{2} \subset C_{* 3} \cup s$ and $C_{3} \subset C_{2}^{*} \cup s$.

From (3.12), $C_{1}$ lies in the closure of $\left(C_{*_{2}} \cap C_{3}{ }^{*}\right) \cup\left(C_{2}^{*} \cap C_{* 3}\right)$. Since $C_{* 2} \cap C_{3}^{*}$ is void and $C_{1} \neq C_{2}, C_{3}$, this implies

$$
C_{1} \subset\left(C_{2}^{*} \cap C_{* 3}\right) \cup s .
$$


Thus $C_{1}$ will separate $C_{* 2} \cap C_{* 3}=C_{* 2}$ and $C_{2}^{*} \cap C_{3}{ }^{*}=C_{3}{ }^{*}$. Replacing $s$ by $v$, we obtain: (3.12)-(3.14) remain valid for $t=u=v$ if $C(v, v, v)$ is interpreted to mean any general osculating circle of $B$ at $v$.

4.2. The following remarks continue the discussions of 3.5. Using 4.1, we do not exclude the possibilities $t=u, u=v, v=e$ or $t=u=v$.

By 3.5, one of the regions (3.13) and (3.14) will lie in $C_{*}(t, u, v)$, the other one in $C^{*}(t, u, v)$. Since $d$ lies in $C^{*}(p, t, v), C^{*}(t, v, e)$ and $C^{*}(t, u, v)$, these relations imply

$$
C^{*}(p, t, v) \cap C^{*}(t, v, e) \subset C^{*}(t, u, v)
$$

and therefore

$$
C_{*}(p, t, v) \cap C_{*}(t, v, e) \subset C_{*}(t, u, v) .
$$

Specializing $t=p$, we obtain

$$
C^{*}(\tau, v) \cap C^{*}(p, v, e) \subset C^{*}(p, u, v)
$$

and

$$
C_{*}(\tau, v) \cap C_{*}(p, v, e) \subset C_{*}(p, u, v) .
$$

Applying the case $v=e$ of (4.3) and (4.4) and replacing afterwards $u$ by $v$, we obtain

$$
\begin{aligned}
& C^{*}(\tau, e) \cap C^{*}(p, e, e) \subset C^{*}(p, v, e), \\
& C_{*}(\tau, e) \cap C_{*}(p, e, e) \subset C_{*}(p, v, e) .
\end{aligned}
$$

We now combine (4.5) with (4.3) and (4.6) with (4.4). This yields on account of (3.10)

$$
\begin{aligned}
C^{*}(\tau, e) \cap C^{*}(p, e, e) & =C^{*}(\tau, e) \cap\left(C^{*}(\tau, e) \cap C^{*}(p, e, e)\right) \\
& \subset C^{*}(\tau, v) \cap C^{*}(p, v, e) \\
& \subset C^{*}(p, u, v)
\end{aligned}
$$

and

$$
\begin{aligned}
C_{*}(p) \cap C_{*}(p, e, e) & \subset C_{*}(p) \cap\left(C_{*}(\tau, e) \cap C_{*}(p, e, e)\right) \\
& \subset C_{*}(\tau, v) \cap C_{*}(p, v, e) \\
& \subset C_{*}(p, u, v) .
\end{aligned}
$$

4.3. The following relation is similar to (4.2):

$$
C_{*}(p, t, u) \cap C_{*}(p, u, v) \subset C_{*}(t, u, v) .
$$

Proof. We assume first that $p, t, u, v$ are mutually distinct. The region $(4.10)$

$$
C_{*}(p, t, u) \cap C_{*}(p, u, v)
$$

is bounded by two arcs of the circles $C(p, t, u)$ and $C(p, u, v)$ with the com- 
mon end points $p$ and $u$. Since $t \subset C^{*}(p, u, v)$ and $v \subset C^{*}(p, t, u)$, these arcs do not contain $t$ and $v$ respectively. Hence they meet $C(t, u, v)$ only at $u$ and the region (4.10) is contained in one of the two regions bounded by $C(t, u, v)$. Since the boundary point $p$ of $(4.10)$ lies in $C_{*}(t, u, v)$, this implies (4.9).

The arguments of 4.1 now show that (4.9) remains valid if the points $p, t, u, v$ cease to be mutually distinct.

By (4.4), (4.9), and (4.2),

$$
\begin{aligned}
C_{*}(\tau, v) \cap C_{*}(p, v, e) & =\left(C_{*}(\tau, v) \cap C_{*}(p, v, e) \cap C_{*}(p, v, e)\right. \\
& \subset C_{*}(p, t, v) \cap C_{*}(p, v, e) \\
& =C_{*}(p, t, v) \cap\left(C_{*}(p, t, v) \cap C_{*}(p, v, e)\right) \\
& \subset C_{*}(p, t, v) \cap C_{*}(t, v, e) \\
& \subset C_{*}(t, u, v) .
\end{aligned}
$$

This relation holds for any choice of $e$ between $v$ and $d$. Letting $e$ tend to $v$, we obtain

$$
C_{*}(\tau, v) \cap C_{*}(p, v, v) \subset C_{*}(t, u, v) .
$$

4.4. Let $o$ denote the pencil of the orthogonal circles of $\tau$. Making $B$ small enough, we may assume that $C(o, d)$ does not meet $B$. By Theorem $3, B \cup p$ satisfies Condition I at $p$. Hence by 1.5 (iii),

$$
\lim _{s \rightarrow p} C(o, s)=p .
$$

Let $v \subset B$. If $s \subset C^{*}(o, v) \cap B$, the points $s$ and $d$ lie on the same side of $C(o, v)$. Since $B$ is connected, $s$ will lie in the region bounded by $C(o, v)$ and $C(o, d)$. Hence $C(o, s)$ will then lie in the union of this region with $p$. Thus (4.12) implies

$$
s \subset C_{*}(0, v)
$$

for every $s$ sufficiently close to $p$. As the circle $C(o, v)$ meets $A_{3}$ not more than twice, this implies that it meets $B$ exactly once at $v$ and nowhere else. Hence (4.13) holds true for every $s \subset B$ between $p$ and $v$.

Suppose the circle $C(t, u, v)$ meets $B$ three times and the points $p, t, u, v, e$ lie on the closure of $B$ in the indicated order. Two or even all of the points $t, u, v$ may coincide.

Since $C(\tau, v)$ meets $C(t, u, v)$, the pencil $\tau$ contains a circle lying in $C_{*}(\tau, v)$ $\cup C(\tau, v)$ and touching $C(t, u, v)$ from within, say at $R$. Thus

$$
\begin{aligned}
& R=C(\tau, R) \cap C(t, u, v), \\
& R \subset C_{*}(\tau, v) \cup C(\tau, v) .
\end{aligned}
$$

The circle $C(o, R)$ can be characterized as the unique circle of $o$ normal to $C(t, u, v)$. We wish to prove the following: 
Lemma. $C(o, R)$ intersects $B$.

By (4.12), $R \subset C^{*}(o, s)$ for every $s$ close to $p$. Hence it suffices to prove (4.16)

$$
R \nsubseteq C^{*}(o, v) \text {. }
$$

Proof of (4.16). Let $s$ move on $B$ between $p$ and $v$. The circle $C(p, s, v)$ meets $C(\tau, v)$ and $C(o, v)$ at $p$ and $v$. By 3.51 and (4.13), $s$ lies in

$$
C_{*}(\tau, v) \cap C_{*}(o, v) .
$$

Hence $C(p, s, v)$ meets this region. The argument of 3.52 now shows that $C(p, s, v)$ does not meet the region

$$
C_{*}(\tau, v) \cap C^{*}(o, v) .
$$

Thus (4.17) lies in either $C_{*}(p, s, v)$ or $C^{*}(p, s, v)$. The region $C_{*}(p, s, v)$ depends continuously on $s$ and tends to $C_{*}(\tau, v)$ if $s$ converges to $p$. Since the region (4.17) lies in $C_{*}(\tau, v)$, it will therefore lie in $C_{*}(p, s, v)$ for every $s$ between $p$ and $v$. Letting $s$ tend to $v$, we obtain

$$
C_{*}(\tau, v) \cap C^{*}(o, v) \subset C_{*}(p, v, v) .
$$

Combining this formula with (4.11), we have

$$
\begin{aligned}
C_{*}(\tau, v) \cap C^{*}(o, v) & =C_{*}(\tau, v) \cap\left[C_{*}(\tau, v) \cap C^{*}(o, v)\right] \\
& \subset C_{*}(\tau, v) \cap C_{*}(p, v, v) \\
& \subset C_{*}(t, u, v) .
\end{aligned}
$$

In particular, $R \subset C(t, u, v)$ implies

$$
R \nsubseteq C_{*}(\tau, v) \cap C^{*}(o, v) .
$$

If $R \subset C_{*}(\tau, v)$, our assertion follows from (4.18). Let $R \nsubseteq C_{*}(\tau, v)$. Then by (4.15), $R \subset C(\tau, v)$ and hence by (4.14)

$$
v \subset C(\tau, v) \cap C(t, u, v)=C(\tau, R) \cap C(t, u, v)=R .
$$

Thus $R=v$ and (4.16) becomes trivial.

\section{Conformally elementary points}

5.1. A point $p$ of an $\operatorname{arc} A$ is conformally elementary if a neighbourhood of $p$ exists on $A$ which is decomposed by $p$ into two one-sided neighbourhoods of order three. By Theorem 3, their closures are strongly differentiable at $p$.

Let $p$ be a differentiable conformally elementary point of an arc $A$ and let $\left(a_{0}, a_{1}, a_{2}\right)$ or $\left(a_{0}, a_{1}, a_{2}\right)_{0}$ be the characteristic of $p$. Then Theorem 1 can be sharpened. We shall prove that $p$ has the cyclic order $a_{0}+a_{1}+a_{2}$. This THEOREM 4 remains valid if a point $q \neq p$ is counted twice on any nonosculating general tangent circle of $q$ and three times on any general osculating circle of $q$ and if $p$ itself is counted $a_{0}\left[a_{0}+a_{1} ; a_{0}+a_{1}+a_{2}\right]$ times on any nontangent circle 
through $p$ [on any nonosculating tangent circle of $p$; on $C(p)$ ].

We may assume that $A$ itself is decomposed by $p$ into two open $\operatorname{arcs} A_{3}$ and $A_{3}^{\prime}$ of order three. Thus the order of $A$ and therefore that of $p$ is not greater than six. By Theorem 2, each point $q \neq p$ of $A$ then satisfies Condition $\mathrm{I}^{\prime}$. The set of the general osculating circles of $q$ is described in 3.42.

5.2. Let $M$ be a neighbourhood of $p$ on $A$. For any circle $D$ let $\mu(D)$ $=\mu(D, M)$ denote the multiplicity with which $D$ meets $M$.

5.21. Suppose the circle $C$ does not pass through the end points of $M$. Then

$$
\mu(D) \equiv \mu(C)(\bmod 2)
$$

for every $D$ sufficiently close to $C$.

Proof. Suppose $C$ meets $M$ at the points $s$ with the multiplicities $\sigma(s)$ and nowhere else. Thus

$$
\mu(C)=\sum_{s} \sigma(s) .
$$

Construct disjoint neighbourhoods $M_{\mathrm{s}}$ in $M$ about the points $s$. The endpoints of $M_{s}$ lie on the same side or on opposite sides of $C$ depending on whether $\sigma(s)$ is even or odd. If $D$ is sufficiently close to $C$, then $D$ will not pass through the end points of $M_{s}$ and they will lie on the same side of $D$ if and only if they lie on the same side of $C$. On the other hand, $D$ will meet $M_{s}$ with an even or odd multiplicity according as its end points lie on the same side or on opposite sides of $D$. Thus $D$ will meet $M_{s}$ with a multiplicity $\rho(s) \equiv \sigma(s)(\bmod 2)$ if $D$ lies sufficiently close to $C$.

If each $M_{8}$ is omitted from the closure of $M$, we obtain a closed set which has no points in common with $C$. Hence, if $D$ is sufficiently close to $C$, this set does not meet $D$ and we have

$$
\mu(D) \equiv \sum_{0} \rho(s) \equiv \sum_{\bullet} \sigma(s) \equiv \mu(C)(\bmod 2)
$$

5.22. We continue the preceding discussion. Let $C \neq C(p)$. Then

$$
\mu(D) \leqq \mu(C)
$$

for every circle $D$ sufficiently close to $C$ unless

$$
a_{0}=a_{1}=1, \quad C \subset \tau \text { and } p \nsubseteq D
$$

[cf. 5.9; for the multiplicity with which $C$ meets $M$ at $p$ cf. 5.1].

Proof. Let $s \subset C \cap M ; s \neq p$. Suppose there is a sequence of circles $D_{\lambda}$ converging to $C$ and a sequence of neighbourhoods $M_{\lambda}$ of $s$ converging to $s$ such that each $D_{\lambda}$ meets $M_{\lambda}$ at least $\rho$ times; $\rho \leqq 3$. Then each $D_{\lambda}$ can be replaced by another circle. which meets $M_{\lambda}$ in not less than $\rho$ distinct points and such that the sequence of the new circles also converges to $C$. Thus $C$ will meet $M$ at least $\rho$ times at $s$; i.e., $\rho \leqq \sigma(s)$. Hence we have: There exists a neighbour- 
hood of $s$ on $M$ which is met not more than $\sigma(s)$ times by every $D$ sufficiently close to $C$.

Let $p \subset C ; C \varangle \tau$. Then $C$ meets $M$ at $p$ with the multiplicity $a_{0}$. On the other hand, by Theorem 3 , there exists a neighbourhood of $p$ which is met not more than twice by any circle sufficiently close to $C$. By 5.21 , we may also assume that $D$ meets this neighbourhood with a multiplicity $\equiv a_{0}(\bmod 2)$. Hence this multiplicity is $\leqq a_{0}$. This proves (5.2) unless $C \subset \tau$.

From now on let $C \subset \tau, C \neq C(p)$. Let $M_{0}=N_{0} \cup p \cup N_{0}^{\prime}$ be a sufficiently small neighbourhood of $p$. Let $D$ be sufficiently close to $C$. If $p \subset D, D \nsubseteq \tau$, then $D$ will meet $N_{0}$ and $N_{0}^{\prime}$ not more than once each. Hence $D$ meets $M_{0}$ with a multiplicity $\leqq a_{0}+2$ and $\equiv a_{0}+a_{1}(\bmod 2)$. Thus this multiplicity is $\leqq a_{0}+a_{1}$. If $D \subset \tau, D$ will not meet $N_{0}$ and $N_{0}^{\prime}$. Thus $D$ then meets $M_{0}$ with the multiplicity $a_{0}+a_{1}$. In either case we obtain (5.2).

Suppose now $p \llbracket D$. Then $D$ will meet $N_{0}$ and $N_{0}^{\prime}$ not more than twice each. Hence $D$ meets $M_{0}$ with a multiplicity $\leqq 4$ and $\equiv a_{0}+a_{1}(\bmod 2)$. This again yields (5.2) unless $a_{0}=a_{1}=1$.

5.3. Let $A=A_{3} \cup p \cup A_{3}^{\prime} ;$ cf. 5.1. There exists a neighbourhood $M_{1}=N_{1} \cup p$ $\cup N_{1}^{\prime}\left[N_{1} \subset A_{3}, N_{1}^{\prime} \subset A_{3}^{\prime}\right]$ such that every tangent circle of $p$ which meets $N_{1} \cup N_{1}^{\prime}$ meets $A_{3} \cup A_{3}^{\prime}$ exactly $a_{2}$ times. In particular, no tangent circle of $p$ meets $M_{1}$ more than $a_{2}$ times outside $p$.

Proof. A circle of $\tau$ meets $A_{3}$ or $A_{3}^{\prime}$ not more than once each. Thus it meets $A_{3} \cup A_{3}^{\prime}$ not more than twice. By 5.21 a circle will meet $A$ with a multiplicity $\equiv a_{0}+a_{1}+a_{2}(\bmod 2)$ if it is sufficiently close to $C(p)$. Hence $C(\tau, t)$ will meet $A_{3} \cup A_{3}^{\prime}$ with a multiplicity $\equiv a_{2}$ if $t$ is close enough to $p$. Such a circle will therefore meet $A_{3} \cup A_{3}^{\prime}$ exactly $a_{2}$ times.

5.4. There exists a neighbourhood $M_{2} \subset M_{1}$ which is met at most $\left(a_{0}+a_{1}\right.$ $\left.+a_{2}\right)$ times by every circle through $p$.

Proof. On account of 5.3, it suffices to consider nontangent circles. Hence it suffices to construct one-sided neighbourhoods $N_{2} \subset N_{1}$ and $N_{2}^{\prime} \subset N_{1}^{\prime}$ of $p$ such that any circle $D$ through $p$ that meets $N_{2}$ or $N_{2}^{\prime}$ twice will meet $M_{1}$ at most $\left(a_{0}+a_{1}+a_{2}\right)$ times.

By (3.20) and (5.1), $N_{2}$ and $N_{2}^{\prime}$ can be chosen so small that any such circle $D$ is so close to $C(p)$ that it meets $M_{1}$ with a multiplicity $\equiv a_{0}+a_{1}+a_{2}$ (mod 2). Since $D$ meets $N_{1}$ and $N_{1}^{\prime}$ not more than twice each, it will meet $M_{1}$ at most $\left(a_{0}+4\right)$ times. This yields our statement if $a_{1}+a_{2}>2$.

Let $a_{1}+a_{2}=2$, i.e., $a_{1}=a_{2}=1$. Let $e$ denote the end point of $N_{1}$ and suppose the points $u, v, e$ lie on $N_{1} \cup e$ in the indicated order. Making $N_{2}^{\prime}$ still smaller, we may assume that it does not meet $C(p, e, e)$ [cf. 4]. Obviously, $N_{2}^{\prime}$ has no points in common with $C(p)$ and $C(\tau, e)$.

We have

$$
N_{1} \subset C^{*}(p) \cap C_{*}(\tau, e) \cap C^{*}(p, e, e) .
$$

Since $p$ has the characteristic $(1,1,1)$ or $(2,1,1)_{0}$, it follows that 


$$
N_{2}^{\prime} \subset C_{*}(p) \cap C_{*}(p, e, e) \text { or } N_{2}^{\prime} \subset C^{*}(\tau, e) \cap C^{*}(p, e, e) .
$$

Hence (4.8) and (4.7) imply that $N_{2}^{\prime}$ lies either in $C_{*}(p, u, v)$ or in $C^{*}(p, u, v)$. Thus $N_{2}^{\prime}$ does not meet $C(p, u, v)$.

Any circle $D$ through $p$ and two points of $N_{2}^{\prime}$ meets $M_{1}$ with a multiplicity $\equiv a_{0}+1+1(\bmod 2)$; i.e., it meets $N_{1} \cup N_{1}^{\prime}$ an even number of times. It meets $N_{1}^{\prime}$ exactly twice. From the above, $D$ cannot meet $N_{1}$ twice. Hence $D$ and $N_{1}$ are disjoint and $D$ meets $M_{1}$ with the total multiplicity $a_{0}+2=a_{0}+a_{1}+a_{2}$.

5.5. We can now prove Theorem 4 if $a_{0}+a_{1}+a_{2}>4$.

It suffices to show that there is a one-sided neighbourhood $N_{3}^{\prime} \subset N_{2}^{\prime}$ of $p$ such that no circle $D$ through three points of $N_{3}^{\prime} \cup_{p}$ meets $M_{1}$ more than $\left(a_{0}+a_{1}+a_{2}\right)$ times. On account of 5.4 , we need only consider circles $D$ which do not pass through $p$.

By (3.21) and (5.1), $N_{3}^{\prime}$ can be chosen such that any $D$ meets $M_{1}$ with a multiplicity $\equiv a_{0}+a_{1}+a_{2}(\bmod 2)$. Since $p \subsetneq D$ and since $D$ meets $N_{1}$ and $N_{1}^{\prime}$ at most three times each it will meet $M_{1}$ at most six times. This yields our assertion.

5.6. The case $a_{0}+a_{1}+a_{2}=4 ; a_{0}=1$. Let $M_{3} \subset M_{2}$ be so small that 4.4 can be applied to $N_{3}=M_{3} \cap N_{2}$ and $N_{3}^{\prime}=M_{3} \cap N_{2}^{\prime}$. Thus some circle of $o$ does not meet $N_{3} \cup N_{3}^{\prime}$. Since $a_{0}=1$, it will intersect $M_{3}$ at $p$. Hence no circle of $o$ can meet both $N_{3}$ and $N_{3}^{\prime}$ and the lemma of 4.4 implies that no circle will meet $N_{3}$ and $N_{3}^{\prime}$ three times each. Taking 5.4 into account, we have: No circle [through $p$ ] meets $M_{3}$ more than five [four] times.

By Theorem 3 and 5.2, a neighbourhood $M_{4} \subset M_{3}$ of $p$ exists such that every circle through three points of $M_{4} \cap N_{3}$ or of $M_{4} \cap N_{3}^{\prime}$ meets $M_{3}$ with an even multiplicity, i.e., four times. Hence $M_{4}$ has the order four.

5.7. The case $(2,1,1)_{0}$. Let $e \subset N_{2}, e^{\prime} \subset N_{2}^{\prime}$. Let $M_{e}$ denote the neighbourhood of $p$ with the end points $e$ and $e^{\prime}$. By 5.3, $C(\tau, e)\left[C\left(\tau, e^{\prime}\right)\right]$ meets $M_{2}$ exactly three times at $p$, just once at $e\left[e^{\prime}\right]$ and nowhere else. By 5.22, any circle through $e\left[e^{\prime}\right]$ sufficiently close to $C(\tau, e)\left[C\left(\tau, e^{\prime}\right)\right]$ meets $M_{2}$ not more than three times near $p$, exactly once at $e\left[e^{\prime}\right]$ and nowhere else, altogether at most four times. Thus by Theorem 3 , there exists a neighbourhood $M_{3} \subset M_{e}$ such that a circle through $e$ or $e^{\prime}$ and two points of $N_{3}=M_{3} \cap N_{2}$ $\left[N_{3}^{\prime}=M_{3} \cap N_{2}^{\prime}\right]$ can meet $M_{2}$ only once more.

Choose $u \subset N_{3}$ and $u^{\prime} \subset N_{3}^{\prime}$ arbitrarily. Let $\pi$ denote the pencil of the circles through $u$ and $u^{\prime}$. By 5.4, $C(\pi, p)$ meets $M_{2}$ only four times. Thus it meets $M_{2}$ exactly twice at $p$, once each at $u$ and $u^{\prime}$ and nowhere else.

Let $t$ lie on $N_{3}$ and sufficiently close to $p$. Then $C(\pi, t)$ meets $M_{2}$ with an even multiplicity $\leqq 4$ and hence exactly four times. With $t$, the fourth point lies close to $p$. Since $C(\pi, p) \nsubseteq \tau$, that fourth point will lie on $N_{2}^{\prime}$ and hence on $N_{3}^{\prime} \quad$ [cf. 5.2 and Theorem 3]. In particular, $e$ and $e^{\prime}$ will not lie on $C(\pi, t)$ and we have

The points $p, e, e^{\prime}$ lie on the same side of $C(\pi, t)$. 
Obviously, (5.3) remains valid if $t$ lies on $N_{3}^{\prime}$ sufficiently close to $p$.

From the above, $C(\pi, p)$ is distinct from $C(\pi, e)$ and $C\left(\pi, e^{\prime}\right)$. Let $\pi_{1}$ denote the set of those circles $C \subset \pi$ such that $p, e, e^{\prime}$ lie on the same side of $C$. Thus

$$
C(\pi, t) \subset \pi_{1}
$$

for every $t$ sufficiently close to $p[t \neq p]$. Hence $\pi_{1}$ is a nonvoid open interval on $\pi$ bounded by $C(\pi, p)$ and either $C(\pi, e)$ or $C\left(\pi, e^{\prime}\right)$. Without loss of generality assume that $C(\pi, e)$ is a boundary circle of $\pi_{1}$.

Suppose $C(\pi, e)$ meets $N_{3}$ twice. By our construction of $M_{3}, C(\pi, e)$ then meets $N_{2}^{\prime} \cup_{p}$ exactly once at $u^{\prime}$ and nowhere else. Hence it separates $p$ and $e^{\prime}$. The same will hold true of any circle of $\pi$ sufficiently close to $C(\pi, e)$. But this is impossible because any neighbourhood of $C(\pi, e)$ will contain circles of $\pi_{1}$. Hence $C(\pi, e)$ meets $N_{3}$ only once.

Let $t$ move on $N_{3}$. By Theorem $2, C(\pi, t)$ depends continuously on $t$ even when $t$ passes through $u$. Since $C(\pi, t)$ meets $N_{3}$ twice, we have $C(\pi, t)$ $\neq C(\pi, e)$. From the above, $C(\pi, t) \neq C(\pi, p)$. Hence (5.4) will remain valid for every $t \subset N_{3}$. This yields (5.3) for every such $t$. Hence $C(\pi, t)$ meets $M_{e} \cap N_{2}\left[M_{e} \cap N_{2}^{\prime}\right]$ with an even multiplicity, i.e., exactly twice. Thus every circle of $\pi$ which meets $N_{3}$ twice will meet $M_{0}$ exactly four times. Hence no circle of $\pi$ can meet $M_{3}$ more than four times. Our construction of $M_{3}$ being independent of $\pi, M_{3}$ therefore has the order four [cf. 5.4].

5.8. The case $a_{0}+a_{1}+a_{2}=3$.

5.81. Suppose the points $p, t, u, v$ lie on $N_{2} \cup p$ in the indicated order. The points $t, u, v$ need not be mutually distinct.

By 5.4, the circles $C(p, t, u)$ and $C(p, u, v)$ meet $M_{2}$ exactly three times and do not meet $N_{2}^{\prime}$. Using on $N_{2}$ the orientation of 3.5 we obtain

$$
N_{2}^{\prime} \subset C_{*}(p, t, u) \cap C_{*}(p, u, v) \text {. }
$$

Hence (4.9) implies $N_{2}^{\prime} \subset C_{*}(t, u, v)$. In particular, $C(t, u, v)$ does not meet $N_{2}^{\prime}$. Symmetrically, any circle through three points of $N_{2}^{\prime}$ does not meet $N_{2}$.

5.82. Let $e \subset N_{2}, e^{\prime} \subset N_{2}^{\prime}$. Let $M_{0}$ denote the neighbourhood of $p$ bounded by $e$ and $e^{\prime}$. By 5.3, $C(\tau, e)\left[C\left(\tau, e^{\prime}\right)\right]$ meets $M_{2}$ exactly twice at $p$, once at $e$ $\left[e^{\prime}\right]$ and nowhere else. Thus by 5.2 , any circle through $e\left[e^{\prime}\right]$ sufficiently close to $C(\tau, e)\left[C\left(\tau, e^{\prime}\right)\right]$ meets $M_{2}$ with an even multiplicity near $p$, exactly once at $e\left[e^{\prime}\right]$ and nowhere else. By 5.81 and 5.4, the order of $M_{2}$ is not greater than four. Hence such a circle meets $M_{2}$ not more than twice near $p$. Theorem 3 therefore implies the existence of a neighbourhood $M_{3} \subset M_{e}$ such that the circles through $e$ or $e^{\prime}$ and two points of $N_{3}=M_{3} \cap N_{2}\left[N_{3}^{\prime}=M_{3} \cap N_{2}^{\prime}\right]$ do not meet $M_{2}$ elsewhere.

Let $u \subset N_{3}, u^{\prime} \subset N_{3}^{\prime}$. By 5.4, $C\left(p, u, u^{\prime}\right)$ meets $M_{2}$ exactly three times. Thus it separates $e$ and $e^{\prime}$. From the above, a circle $C\left(t, u, u^{\prime}\right)$ does not pass through $e$ or $e^{\prime}$ if $t \subset N_{8} \cup N_{3}^{\prime}$. Let $t$ move on $M_{3}$. Then the circle $C\left(t, u, u^{\prime}\right)$ depends continuously on $t$ [cf. Theorem 2]. Thus it always separates $e$ and $e^{\prime}$. 
Hence it meets $M_{\theta}$ an odd number of times. Since $M_{\theta} \subset M_{2}$, the order of $M_{0}$ is not greater than four. Thus $C\left(t, u, u^{\prime}\right)$ meets $M_{6}$ and hence also $M_{3}$ only three times. This implies that $M_{3}$ has the order three.

5.9. The following remarks may be of interest. We omit their proofs as no new methods are involved.

THEOREM 5. Let $p$ be a conformally elementary point on the arc $A$. Then

(i) p satisfies Condition $\mathrm{I}^{\prime}$ if and only if it satisfies Condition I and $a_{0}=1$.

(ii) $A$ is strongly differentiable at $p$ if and only if it is differentiable at $p$ and $a_{0}=a_{1}=1$.

We note that $a_{0}$ is defined if Condition I is satisfied; cf. 1.5 (ii). The case $a_{2}=2$ of part (ii) can be verified by means of an argument similar to that leading to (3.19).

Formula (5.2) can now readily be extended.

Given an $\operatorname{arc} A$ divided into two open arcs of order three by the differentiable point $p$. Let $\mu(D)$ denote the multiplicity with which a circle $D$ meets $A$. We then have the

Corollary. Suppose the circle $C$ does not pass through the end points of $A$. Then $\mu(D) \leqq \mu(C)$ for every $D$ sufficiently close to $C$.

\title{
REFERENCES
}

1. F. Denk and O. Haupt, Über die Singularitaten reeller Bogen im $R_{n}$, J. Reine Angew. Math. vol. 183 (1941) pp. 69-91.

2. J. Hjelmslev, Introduction a la theorie des suites monotones, Oversigt Kgl. Danske Vidensk. Selsk. Forh., No. 1, 1914.

3. N. Lane and P. Scherk, Differentiable points in the conformal plane, Canadian Journal of Mathematics vol. 5 (1953) pp. 512-518.

4. A. Marchaud, Sur les continus d'ordre borné, Acta Math. vol. 55 (1930) pp. 67-115.

5. P. Scherk, Über differenzierbare Kurven und Bögen I. Zum Begriff der Charakteristik, Casopis pro Pěstování Matematiky a Fysiky vol. 66 (1937) pp. 165-171.

\author{
MCMASTER UNIVERSITY, \\ HaMilton, ONT. \\ UNIVERSITY OF SASKATCHEWAN, \\ SASKatoon, Sask.
}

